\title{
BMJ Open Protocol for a multicentre, prospective, observational cohort study of variation in practice in perioperative analgesic strategies in elective laparoscopic colorectal surgery: the LapCoGesic Study
}

Northern Surgical Trainees Research Association (NoSTRA), Phillippa Burnell, Rachael Coates, Steven Dixon, Lucy Grant, Matthew Grey, Ben Griffiths, Mike Jones, Anantha Madhavan, Iain McCallum, Ross McClean, Karen Naru, Lydia Newton, Paul O'Loughlin, Fadlo Shaban, Anisha Sukha, Sameer Somnath, Syed Shumon, Deena Harji

To cite: Northern Surgical Trainees Research Association (NoSTRA), Burnell P, Coates R, Dixon S, et al. Protocol for a multicentre, prospective, observational cohort study of variation in practice in perioperative analgesic strategies in elective laparoscopic colorectal surgery: the LapCoGesic Study. BMJ Open 2016;6: e008810. doi:10.1136/ bmjopen-2015-008810

- Prepublication history for this paper is available online. To view these files please visit the journal online (http://dx.doi.org/10.1136/ bmjopen-2015-008810).

Received 15 November 2015 Revised 28 January 2016 Accepted 1 February 2016

\section{CrossMark}

Department of Academic Surgery, University of Newcastle, Newcastle upon Tyne, UK

Correspondence to Deena Harji; deena_harji@ hotmail.com

\section{ABSTRACT}

Introduction: Laparoscopic surgery combined with enhanced recovery programmes has become the gold standard in the elective management of colorectal disease. However, there is no consensus with regard to the optimal perioperative analgesic regime in this cohort of patients, with a number of options available, including thoracic epidural spinal analgesia, patientcontrolled analgesia, subcutaneous and/or intraperitoneal local anaesthetics, local anaesthetic wound infiltration catheters and transversus abdominis plane blocks. This study aims to explore any differences in analgesic strategies employed across the North East of England and to assess whether any variation in practice has an impact on clinical outcomes.

Methods and analysis: All North East Colorectal units will be recruited for participation by the Northern Surgical Trainees Research Association (NoSTRA). Data will be collected over a consecutive 2-month period. Outcome measures will include postoperative pain score, postoperative opioid analgesic use and side effects, length of stay, 30-day complication rates, 30day reoperative rates and 30-day readmission rates. Ethics and dissemination: Ethical approval for this study has been granted by the National Research Ethics Service. The protocol will be disseminated through NoSTRA. Individual unit data will be presented at local meetings. Overall collective data will be published in peer-reviewed journals and presented at relevant surgical meetings.

\section{INTRODUCTION}

In recent times, patient outcomes have been vastly improved in colorectal surgery due to
Strengths and limitations of this study

- A multicentre, prospective study with involvement from anaesthetists and surgeons.

- Capture of data from all hospitals across the North East.

- Ability to capture data from patients presenting to other hospitals within the region.

- No quality assurance of perioperative analgesic strategy employed.

- No quality assurance of standard of laparoscopic surgery.

the widespread adoption of laparoscopic surgery. ${ }^{1}$ The reduced surgical stress response observed in laparoscopic surgery translates clinically into reduced postoperative pain, earlier return of gastrointestinal function and reduced length of hospital stay. ${ }^{1-6}$ The quest to maintain perioperative physiological status and reduce surgical stress has led to the development and employment of enhanced recovery programmes. ${ }^{7-10}$ The core principles of enhanced recovery programmes focus on reducing surgical stress by maintaining postoperative physiological function and enhanced mobilisation following surgery. Key to the success of such enhanced recovery programmes is the use of an optimal analgesic regime which ensures adequate pain relief, enables early mobilisation and the early return of gastrointestinal function combined with a low side effect profile.

Early enhanced recovery programmes were developed to be used in open colorectal 
surgery and recommended the use of thoracic epidural analgesia. ${ }^{11}{ }^{12}$ However, applying the same analgesic principles in the laparoscopic setting has not produced the same results in this cohort of patients, with reports of longer length of stay and delayed return of gastrointestinal function associated with the use of epidural analgesia. ${ }^{13-15}$ This has led to a number of alternative analgesic strategies being employed in laparoscopic colorectal surgery, including the use of spinal analgesia, ${ }^{15-19}$ patient-controlled analgesia (PCA), ${ }^{20-22}$ subcutaneous and/or intraperitoneal local anaesthetics, local anaesthetic wound infiltration catheters ${ }^{23} 24$ and transversus abdominis plane (TAP) blocks. ${ }^{25-28}$ There have been a number of cohort studies and randomised controlled trials comparing different analgesic modalities with encouraging results; however, these studies are often single-armed or double-armed studies, comparing only one or two analgesic modalities. Other shortcomings of these studies are that they are often retrospective and single-centre studies, thus making it difficult to generalise the results. Consequently, there remains a lack of consensus on the optimal analgesic strategy in this cohort of patients, ${ }^{11} 29$ thus leading to colorectal units employing analgesic regimes based on individual expertise and experience. The LapCoGesic Study aims to explore differences in analgesic strategies employed across the north-east region in patients undergoing elective laparoscopic colorectal surgery and to assess whether this variation in practice has an impact on clinical outcomes.

\section{METHODS AND ANALYSIS}

\section{Study objectives}

To explore differences in analgesic strategies employed across the north-east region in patients undergoing elective laparoscopic colorectal surgery and to assess whether variation in practice has an impact on clinical and patient-reported outcomes.

\section{Study design}

We aim to undertake a prospective, multicentre, observational cohort study of consecutive patients undergoing elective laparoscopic colorectal surgery, which will be led by the trainee research collaborative.

\section{Setting}

This study will take place across colorectal units across the North East of England over a consecutive period of 2 months. This region has the highest rate of elective laparoscopic resections for primary colorectal cancer in the UK according to the National Bowel Cancer Audit Report 2014. ${ }^{30}$

\section{Recruitment}

All patients undergoing an elective laparoscopic colorectal resection in the North East of England will be recruited into the study. According to the National Bowel Cancer Audit 2014, 1257 patients underwent elective surgery for primary colorectal cancer in the North East of England over a 12-month period. ${ }^{31}$ Based on these figures, minimum expected recruitment would be $\sim 150$ patients from 13 centres across the North East of England.

\section{ELIGIBILITY CRITERIA \\ Inclusion criteria}

All adult patients (aged $>18$ years) undergoing an elective laparoscopic (multiport or single-port) colorectal resection will be included in this study.

\section{Table 1 Secondary outcome measures}

\begin{tabular}{|c|c|}
\hline $\begin{array}{l}\text { Intraoperative analgesic } \\
\text { regime }\end{array}$ & $\begin{array}{l}\text { Intravenous analgesia type and dose } \\
\text { Paracetamol, fentanyl, alfentanil, remifentanil, morphine } \\
\text { Spinal analgesia type and dose } \\
\text { Bupivacaine, fentanyl, morphine, diamorphine } \\
\text { Epidural analgesia type and dose } \\
\text { Bupivacaine and fentanyl infusion } \\
\text { TAP blocks } \\
\text { Local anaesthesia type and dose } \\
\text { Local anaesthesia wound infiltration } \\
\text { Type and dose }\end{array}$ \\
\hline $\begin{array}{l}\text { Postoperative analgesic } \\
\text { regime }\end{array}$ & $\begin{array}{l}\text { Paracetamol, non-steroidal analgesia, morphine patient-controlled analgesia, epidural analgesia, } \\
\text { TAP blocks }\end{array}$ \\
\hline Postoperative pain scores & $\begin{array}{l}\text { Standardised pain scores will be collected as per the pain scales outlined on the National Early } \\
\text { Warning Score charts. This pain scale is a visual analogue scale based on a scale of } 0-10 \text {. }\end{array}$ \\
\hline Postoperative opioid use & $\begin{array}{l}\text { The postoperative oral morphine equivalent dose will be calculated for each patient on a daily } \\
\text { basis up to } 7 \text { days postoperatively or day of discharge if this is earlier. }\end{array}$ \\
\hline Length of stay & $\begin{array}{l}\text { Postoperative HDU/ICU stay will be calculated. Total length of hospital stay will also be } \\
\text { calculated from date of admission to date of discharge. }\end{array}$ \\
\hline 30-day complication rates & $\begin{array}{l}\text { All-cause postoperative morbidity will be calculated as per the Clavien-Dindo classification. This } \\
\text { will also include calculating } 30 \text {-day reoperation rates. }\end{array}$ \\
\hline 30-day reoperation rates & All-cause readmission within the first 30 days postoperatively will be calculated. \\
\hline
\end{tabular}


Table 2 Data fields

Patient age (whole years)
Patient gender
BMI
BMI category

ASA grade

Admission date

Operation date

Primary operative indication

Primary operation type

\section{Surgeon grade}

Anaesthetist grade

Intraoperative analgesia

Intraoperative antiemetic use

Intraoperative complication

\section{Conversion to open}

Duration of operation (whole minutes)

Blood loss

Extraction site size (cm)

Postoperative: ERAS pathway used

Postoperative: Acute Pain Service involved

Postoperative analgesia

Postoperative antiemetic use

Day 1 postoperative lowest respiratory rate

Day 1 postoperative lowest sedation score

Postoperative pain scores at 24 and 48 hours and daily

until 7 days postoperatively or discharge if before 7 days

Postoperative opioid analgesic use at 24 and 48 hours and

daily until 7 days postoperatively or discharge if before

7 days

ITU discharge date

HDU discharge date

Date fit for discharge

Date actual discharged

Complications 30-day postoperatively

Complication type

Surgical complication grade (Clavien-Dindo classification)

Readmission 30 days post discharge

Reoperation 30 days postoperatively

ASA, American Society of Anaesthesiologists; AVPU, alert, voice, pain, unresponsive; BMI, body mass index; CCT, Chicago Community Trust; ERAS, Enhanced Recovery After Surgery; HDU, high dependency unit; ITU, intensive therapy unit; LA, local anaesthetic; NSAIDs, non-steroidal anti-inflammatory drugs; PCA, patient-controlled analgesia; TAP, transversus abdominis plane.
Years

Male, female

$\mathrm{BMI}$ in $\mathrm{kg} / \mathrm{m}^{2}$

Underweight $<18 \mathrm{~kg} / \mathrm{m}^{2}$

Normal $18-24.9 \mathrm{~kg} / \mathrm{m}^{2}$

Overweight $25-29.9 \mathrm{~kg} / \mathrm{m}^{2}$

Moderate obesity $30-34.9 \mathrm{~kg} / \mathrm{m}^{2}$

Severe obesity $35-39.9 \mathrm{~kg} / \mathrm{m}^{2}$

Very severe obesity $>40 \mathrm{~kg} / \mathrm{m}^{2}$

I: A normal healthy patientll: A patient with mild systemic diseaselll: A patient with severe systemic diseaselV: A patient with severe systemic disease, ie, a constant threat to lifeV: A moribund patient who is not expected to survive without the operation $\mathrm{DD} / \mathrm{MM} / \mathrm{YYY}$

DD/MM/YYY

Colorectal malignancy, ulcerative colitis, Crohn's disease, diverticular disease, other (free text)

Right hemicolectomy, left hemicolectomy, sigmoid colectomy, Hartmann's procedure, subtotal colectomy, anterior resection tileostomy, panproctocolectomy, abdominoperineal excision of the rectum

Consultant, registrar (ST3-8), non-training grade, post-CCT fellow Consultant, registrar (ST3-8), non-training grade, post-CCT fellow, core anaesthetic trainee (CT1-2)

Intravenous: paracetamol, NSAIDs, fentanyl, alfentanil, morphine, remifentanil infusion, other

Spinal Anaesthesia: Bupivacaine, Fentanyl, Morphine,

Diamorphine

TAP blocks

LA infiltration

Epidural anaesthesia: bupivacaine and fentanyl, levobupivacaine Ondansetron, cyclizine, dexamethasone, droperidol, other No intraoperative complication, visceral injury, small bowel injury, colonic injury, ureteric injury, bladder injury, vascular injury, other injury (free text)

Yes, no

Minutes

Millilitres

Total size of extraction site wound in centimetres

Yes, no

Yes, no

Paracetamol, NSAIDs, morphine PCA, epidural, TAP blocks, other Ondanestron, cyclizine, other

Breaths/min

AVPU Score

$0-10$

\section{Milligrams}

\section{DD/MM/YYYY \\ DD/MM/YYYY \\ DD/MM/YYYY \\ DD/MM/YYYY \\ Yes, no \\ Free text}

None, I, II, III, IV, V

Yes, no

Yes, no 


\section{Exclusion criteria}

Patients undergoing emergency surgery, a diagnostic laparoscopy, or with a pre-existing chronic pain or fatigue syndromes, chronic opioid use and cognitive impairment will be excluded from the study.

\section{Outcome measures}

The primary outcome measure is postoperative pain scores at 24 hours. This is the recommended outcome measure by the Royal College of Anaesthetists to assess the efficacy of postoperative analgesia. ${ }^{32}$ Secondary outcome measures will include postoperative opioid analgesic use, total length of stay, 30-day postoperative complication rates, complication grade according to the Clavien-Dindo classification, and 30-day reoperative and readmission rates (table 1 ).

\section{Data collection and data management}

Each participating local hospital will be responsible for identifying potentially eligible patients for study recruitment. The principal investigator team will consist of a consultant surgeon, consultant anaesthetist and two trainees from surgery or anaesthesia. Patients will be identified from three clinical areas-outpatient clinic, preoperative assessment clinic and daily elective operating lists-to ensure all potentially eligible patients are captured.

A standardised data collection spreadsheet (Excel 2010; Microsoft, Redmond, Washington, USA) will be used at each centre with predefined data fields. Following completion of the study, all anonymised data will be submitted centrally via a secure, passwordprotected website. The required anonymous data fields of this spreadsheet are shown in table 2. All anonymised data will be subsequently analysed. Outcome data specific to each surgeon who participates in the study will not be collected or analysed.

\section{Statistical analysis}

The results of this study will be prepared in accordance with guidelines set by the Strengthening the Reporting of Observational Studies in Epidemiology (STROBE) statement for observational studies. ${ }^{33}$

Data will be collected and analysed in clinically relevant categories with $\chi^{2}$ or Mann-Whitney $\mathrm{U}$ tests employed to detect differences between groups. Multivariable binary logistic regression will be used to test the influence of clinically plausible variables (ie, analgesic regime, operation type, operative indication, etc) on the outcome measures, to produce adjusted OR and $95 \%$ CI. Excel 2010 will be used for data handling, and statistical modelling in SPSS V.22. Statistical significance is defined as $p \leq 0.05$ in all analyses.

\section{Ethical approval}

Ethical approval for this study has been obtained from the National Research Ethics Committee (REC number $15 / \mathrm{NE} / 0110)$. As this study does not impact on clinical care, individual patient consent will not be sought. Local ethical approval will be sought at each participating centre.

\section{Dissemination}

The protocol will be disseminated through the Northern Surgical Trainees Research Association (NoSTRA). All protocol documents and relevant clinical toolkits will be made available through the NoSTRA website (http:// www.nostragroup.co.uk/projects/lapcogesic). Individual unit data will be presented at local meetings. Overall collective data will be published in peer-reviewed journals and presented at relevant surgical meetings. It is anticipated the results from this prospective study will help inform ongoing clinical research and will be used to inform commissioning and implement changes within the National Health Service (NHS).

\section{DISCUSSION}

Currently, in the UK, there is no agreed consensus on the optimal perioperative analgesic strategy in patients undergoing elective laparoscopic colorectal resections. A prospective survey carried out by the Enhanced Recovery After Surgery (ERAS) society in 2013 identified there was huge variation in current clinical practice with regard to optimal analgesic modality in this cohort of patients. ${ }^{34}$ However, the limitations of this study include the small sample size and the large number of anaesthetists surveyed. Our study is novel in that it investigates current analgesic strategy employed and its subsequent impact on clinical and patient-reported outcomes with collaborative support from surgeons and anaesthetists. The data generated from this prospective, multicentre, observational cohort study will help identify and plan future areas of research, to evaluate the efficacy of multimodal analgesic regimes in elective laparoscopic colorectal practice, to develop a consensus over appropriate clinical end points, to accumulate data for generation of power calculations, to qualitatively analyse patient, surgeon and anaesthetic values and opinions, to achieve consensus on a trial question and its target population, with an overall aim to inform the design of a phase III randomised controlled trial.

Twitter Follow Deena Harji at @nostragroup

Collaborators Northern Surgical Trainees Research Association, PB, RC, SD, LG, MG, BG, DH, MJ, AM, IM, RM, KN, LN, PO, FS, AS, SSo and SSh.

Contributors All authors have equally contributed to the writing and development of this manuscript. DH, BG, SSo, KN, PO and IM contributed to study concept, design and development. DH, SSh, LN, PB, RC and AM were responsible for manuscript writing. $\mathrm{DH}, \mathrm{RM}$ and $\mathrm{MJ}$ were responsible for data analysis and statistical input. MJ, FS, AS, MG, LG and SD were responsible for data acquisition. BG, PO, SSo and KN were responsible for final manuscript approval.

Competing interests None declared.

Ethics approval National Research Ethics Committee-York.

Provenance and peer review Not commissioned; externally peer reviewed. 
Data sharing statement The data amassed from this study will be available to the NoSTRA collaborative.

Open Access This is an Open Access article distributed in accordance with the Creative Commons Attribution Non Commercial (CC BY-NC 4.0) license, which permits others to distribute, remix, adapt, build upon this work noncommercially, and license their derivative works on different terms, provided the original work is properly cited and the use is non-commercial. See: http:// creativecommons.org/licenses/by-nc/4.0/

\section{REFERENCES}

1. Guillou PJ, Quirke P, Thorpe $\mathrm{H}$, et al. Short-term endpoints of conventional versus laparoscopic-assisted surgery in patients with colorectal cancer (MRC CLASICC trial): multicentre, randomised controlled trial. Lancet 2005;365:1718-26.

2. Veldkamp R, Kuhry E, Hop WC, et al. Laparoscopic surgery versus open surgery for colon cancer: short-term outcomes of a randomised trial. Lancet Oncol 2005;6:477-84

3. Hazebroek EJ, Color Study G. COLOR: a randomized clinical trial comparing laparoscopic and open resection for colon cancer. Surg Endosc 2002;16:949-53.

4. Nishiguchi K, Okuda J, Toyoda M, et al. Comparative evaluation of surgical stress of laparoscopic and open surgeries for colorectal carcinoma. Dis Colon Rectum 2001;44:223-30.

5. Wichmann MW, Huttl TP, Winter $\mathrm{H}$, et al. Immunological effects of laparoscopic vs open colorectal surgery: a prospective clinical study Arch Surg 2005;140:692-7.

6. Braga M, Vignali A, Zuliani W, et al. Metabolic and functional results after laparoscopic colorectal surgery: a randomized, controlled trial. Dis Colon Rectum 2002;45:1070-7.

7. Wilmore DW, Kehlet H. Management of patients in fast track surgery. BMJ 2001;322:473-6.

8. Fearon KC, Ljungqvist $\mathrm{O}$, Von Meyenfeldt M, et al. Enhanced Recovery After Surgery: a consensus review of clinical care for patients undergoing colonic resection. Clin Nutr 2005;24:466-77.

9. Kehlet H. Fast-track colorectal surgery. Lancet 2008;371:791-3.

10. Kehlet $\mathrm{H}$, Wilmore DW. Evidence-based surgical care and the evolution of fast-track surgery. Ann Surg 2008;248:189-98.

11. Nygren J, Thacker J, Carli F, et al. Guidelines for perioperative care in elective rectal/pelvic surgery: Enhanced Recovery After Surgery (ERAS $((\mathrm{R}))$ ) Society recommendations. World J Surg 2013;37:285-305

12. Gustafsson UO, Scott MJ, Schwenk W, et al. Guidelines for perioperative care in elective colonic surgery: Enhanced Recovery After Surgery (ERAS(R)) Society recommendations. Clin Nutr 2012;31:783-800.

13. Halabi WJ, Kang CY, Nguyen VQ, et al. Epidural analgesia in laparoscopic colorectal surgery: a nationwide analysis of use and outcomes. JAMA Surg 2014;149:130-6.

14. Halabi WJ, Jafari MD, Nguyen $V Q$, et al. A nationwide analysis of the use and outcomes of epidural analgesia in open colorectal surgery. J Gastrointest Surg 2013;17:1130-7.

15. Day A, Smith $R$, Jourdan I, et al. Retrospective analysis of the effect of postoperative analgesia on survival in patients after laparoscopic resection of colorectal cancer. $\mathrm{Br} J$ Anaesth 2012;109:185-90.

16. Levy BF, Scott MJ, Fawcett W, et al. Randomized clinical trial of epidural, spinal or patient-controlled analgesia for patients undergoing laparoscopic colorectal surgery. Br J Surg 2011;98:1068-78.
17. Virlos I, Clements D, Beynon J, et al. Short-term outcomes with intrathecal versus epidural analgesia in laparoscopic colorectal surgery. Br J Surg 2010;97:1401-6.

18. Kong SK, Onsiong SM, Chiu WK, et al. Use of intrathecal morphine for postoperative pain relief after elective laparoscopic colorectal surgery. Anaesthesia 2002;57:1168-73.

19. Peravali R, Brock R, Bright E, et al. Enhancing the Enhanced Recovery Program in Colorectal Surgery-Use of Extended-Release Epidural Morphine (DepoDur(R)). Ann Coloproctol 2014;30:186-91.

20. Hubner M, Blanc C, Roulin D, et al. Randomized clinical trial on epidural versus patient-controlled analgesia for laparoscopic colorectal surgery within an enhanced recovery pathway. Ann Surg 2015;261:648-53

21. Kaminski JP, Pai A, Ailabouni L, et al. Role of epidural and patient-controlled analgesia in site-specific laparoscopic colorectal surgery. JSLS 2014;18:pii: e2014.00207.

22. Senagore AJ, Delaney CP, Mekhail N, et al. Randomized clinical trial comparing epidural anaesthesia and patient-controlled analgesia after laparoscopic segmental colectomy. Br J Surg 2003;90:1195-9.

23. Boulind $\mathrm{CE}$, Ewings $\mathrm{P}$, Bulley $\mathrm{SH}$, et al. Feasibility study of analgesia via epidural versus continuous wound infusion after laparoscopic colorectal resection. Br J Surg 2013;100:395-402.

24. Moore SE, Reid KC, Draganic BD, et al. Randomized clinical trial of ropivacaine wound infusion following laparoscopic colorectal surgery. Tech Coloproctol 2012;16:431-6.

25. Niraj G, Kelkar A, Hart E, et al. Comparison of analgesic efficacy of four-quadrant transversus abdominis plane (TAP) block and continuous posterior TAP analgesia with epidural analgesia in patients undergoing laparoscopic colorectal surgery: an open-label, randomised, non-inferiority trial. Anaesthesia 2014;69:348-55.

26. Keller DS, Ermlich BO, Delaney CP. Demonstrating the benefits of transversus abdominis plane blocks on patient outcomes in laparoscopic colorectal surgery: review of 200 consecutive cases. J Am Coll Surg 2014;219:1143-8.

27. Walter CJ, Maxwell-Armstrong C, Pinkney TD, et al. A randomised controlled trial of the efficacy of ultrasound-guided transversus abdominis plane (TAP) block in laparoscopic colorectal surgery. Surg Endosc 2013;27:2366-72.

28. Favuzza J, Brady K, Delaney CP. Transversus abdominis plane blocks and enhanced recovery pathways: making the 23-h hospital stay a realistic goal after laparoscopic colorectal surgery. Surg Endosc 2013;27:2481-6.

29. Gustafsson UO, Scott MJ, Schwenk W, et al. Guidelines for perioperative care in elective colonic surgery: Enhanced Recovery After Surgery (ERAS((R))) Society recommendations. World J Surg 2013;37:259-84.

30. AoCoGBa. National Bowel Cancer Audit Progress Report 2014. Ireland, 2014.

31. Muhammad A, Lamendola O, Daas A, et al. Association between colonic diverticulosis and prevalence of colorectal polyps. Int $J$ Colorectal Dis 2014;29:947-51.

32. Royal College of Anaesthetists. Efficacy of acute pain management in the postoperative period. In. 2014. https://www.rcoa.ac.uk/system/ files/CSQ-ARB-section11.pdf

33. von Elm E, Altman DG, Egger M, et al. The Strengthening the Reporting of Observational Studies in Epidemiology (STROBE) statement: guidelines for reporting observational studies. Lancet 2007;370:1453-7.

34. UK Enhanced Recovery After Surgery Society. Post-Operative Analgesia after Colorectal Surgery in ERAS. An online survey of ERAS UK members. In. 2013. http://www.erasuk.net/uploads/2/6/4/ 0/26401678/session_1 report on eras uk survey j foster.pdf 
Correction: Protocol for a multicentre, prospective, observational cohort study of variation in practice in perioperative analgesic strategies in elective laparoscopic colorectal surgery: the LapCoGesic Study

Northern Surgical Trainees Research Association (NoSTRA), Burnell P, Coates R, et $a l$. Protocol for a multicentre, prospective, observational cohort study of variation in practice in perioperative analgesic strategies in elective laparoscopic colorectal surgery: the LapCoGesic Study. BMJ Open 2016;6:e008810. doi: 10.1136/bmjopen-2015-008810

The author name 'Matthew Grey' is misspelled and should be 'Matthew Gray'.

Open Access This is an Open Access article distributed in accordance with the Creative Commons Attribution Non Commercial (CC BY-NC 4.0) license, which permits others to distribute, remix, adapt, build upon this work non-commercially, and license their derivative works on different terms, provided the original work is properly cited and the use is non-commercial. See: http://creativecommons.org/licenses/by-nc/4.0/

C Article author(s) (or their employer(s) unless otherwise stated in the text of the article) 2017. All rights reserved. No commercial use is permitted unless otherwise expressly granted.

BMJ Open 2017;7:e008810corr1. doi:10.1136/bmjopen-2015-008810corr1 